\title{
Psychometric evaluation of the family caregiver ICU delirium knowledge questionnaire
}

\author{
Karla D. Krewulak ${ }^{1}$, Margaret J. Bull ${ }^{2}$, E. Wesley Ely ${ }^{3}$, Henry T. Stelfox ${ }^{1,4}$ and Kirsten M. Fiest ${ }^{1,4,5^{*}}$
}

\begin{abstract}
Background: Delirium is a common condition in critically ill patients, affecting nearly half of all patients admitted to an intensive care unit (ICU). Family caregivers of critically ill patients can be partners in the early recognition, prevention and management of delirium provided they are aware of the signs/symptoms and appropriate nonpharmacological strategies that might be taken. Valid, reliable instruments that assess family caregiver knowledge are essential so that nurses can prepare family caregivers to be effective partners. The purpose of the current study was to (a) adapt an existing caregiver delirium knowledge questionnaire (CDKQ) for use by nurses to measure a family caregiver's delirium knowledge in the ICU; and (b) examine the psychometric properties and structure of the adapted Caregiver ICU Delirium Knowledge Questionnaire (CIDKQ).

Methods: In this cross-sectional study, a multidisciplinary team developed the 21-item CIDKQ (possible score range: 0-21) and administered it to 158 family caregivers of critically ill patients. Descriptive statistics were examined for all variables. The CIDKQ was analyzed for face validity, content validity, reliability and internal consistency.

Results: The mean CIDKQ score was 14.1 (SD: 3.5, range $=2$ to 21). Path analysis revealed that a family caregiver's delirium knowledge in the actions and symptoms dimensions had a direct effect on knowledge of delirium risk factors. The CIDKQ was found to have face validity and reliability (Cronbach's $a=0.79$ ).
\end{abstract}

Conclusions: The findings indicated good validity and reliability of the CIDKQ as a measure of ICU delirium knowledge in family caregivers of critically ill patients.

Keywords: Delirium prevention and management, Family caregivers, Critical care, Intensive care unit

\section{Background}

Delirium is a common neuropsychiatric complication in the ICU that affects over $50 \%$ of critically ill patients [13 ] and is associated with negative short-term (e.g., longer ICU stay, increased ICU and in-hospital mortality) and long-term outcomes (e.g., post-intensive care syndrome, (PICS)) [4]. Family caregivers often experience distress from witnessing delirium in their loved ones [5-8], which can lead to the development of adverse psychological outcomes after ICU discharge known as PICS-Family [9-11]. The Society of Critical Care Medicine guidelines for family-centred

\footnotetext{
* Correspondence: kmfiest@ucalgary.ca

'Department of Critical Care Medicine, Alberta Health Services \& University of Calgary, Calgary, AB, Canada

${ }^{4}$ Department of Community Health Sciences and O'Brien Institute for Public Health, University of Calgary, Calgary, AB, Canada

Full list of author information is available at the end of the article
}

care in the ICU [12] suggest providing family education programs and including family caregivers in patient care has the potential to improve family caregiver-centred outcomes [10, 12]. Current delirium guidelines recommend multicomponent non-pharmacological interventions such as a reorientation protocol (eyeglass/hearing optimization, orientation of day/time/location), environment protocol (improve sleep) or mobility have the potential to prevent or reduce the number of delirium days [13]. These nonpharmacological interventions can be employed by family caregivers, providing an opportunity for family caregivers to be engaged in patient care.

Many family caregivers do not possess sufficient delirium knowledge to function as partners in the prevention and management of delirium using non-pharmacological strategies. A valid, reliable caregiver delirium knowledge 
questionnaire (CDKQ) to help nurses evaluate a family caregivers' delirium knowledge exists, but was validated in community-dwelling family caregivers of elective knee and hip patients [14]. There are additional risk factors that predispose a patient to develop delirium in the ICU (e.g., severity of illness, mechanical ventilation, use of benzodiazepines) $[13,15]$ compared to that of a patient in the hospital or community. To our knowledge, a valid, reliable measure of family caregiver knowledge of ICU delirium does not exist in the literature. The purpose of this study was to (a) adapt the existing CDKQ for use in family caregivers of critically ill patients in the ICU and (b) establish the validity and reliability of this Caregiver ICU Delirium Knowledge Questionnaire (CIDKQ). In turn, nurses can use the CIDKQ to specifically target learning needs of family caregivers and better prepare them to partner in the prevention and management of delirium. This might improve patient- and family-centred outcomes.

\section{Methods}

\section{Instrument refinement}

The CDKQ questions were reworded to be ICU-specific. After reviewing relevant literature on ICU delirium risk factors and outcomes, one additional item (increased delirium risk in patients who are mechanically ventilated) was added to the CIDKQ $[13,15,16]$. Similar to the CDKQ
[14], the Symptom Interpretation Model (SIM) framework was used [17], which includes three dimensions of delirium knowledge: risks, action and symptoms. A total of $21 \mathrm{mul}-$ tiple choice (yes/no/don't know) items were included in the CIDKQ addressing three dimensions of delirium knowledge: risk factors (items 1-10), actions (items 11-16) and symptoms (items 17-21). Any correct answer is coded as 1 and any incorrect answer (including "don't know") as 0.

\section{Design and sample}

The study was approved by the Conjoint Health Research Ethics Board at the University of Calgary (Reference number: REB18-0331). To validate the CIDKQ, we performed a cross-sectional study between June 19, 2018 and July 31, 2019 using a convenience sample of family caregivers from the 28-bed general systems adult ICU and the family waiting rooms for the 16-bed cardiovascular ICU (CV-ICU) at Foothills Medical Centre in Calgary, Canada (catchment population: 1.5 million). The general systems adult ICU includes neurological, medical, surgical and long-term ICU patients. Upon ICU admission, patients and their families are provided with an ICU delirium pamphlet as part of a welcome package. The CV-ICU includes patients who are pre- and postcardiovascular surgery. Bedside nurses screen for delirium once per shift (twice daily) in both the ICU and

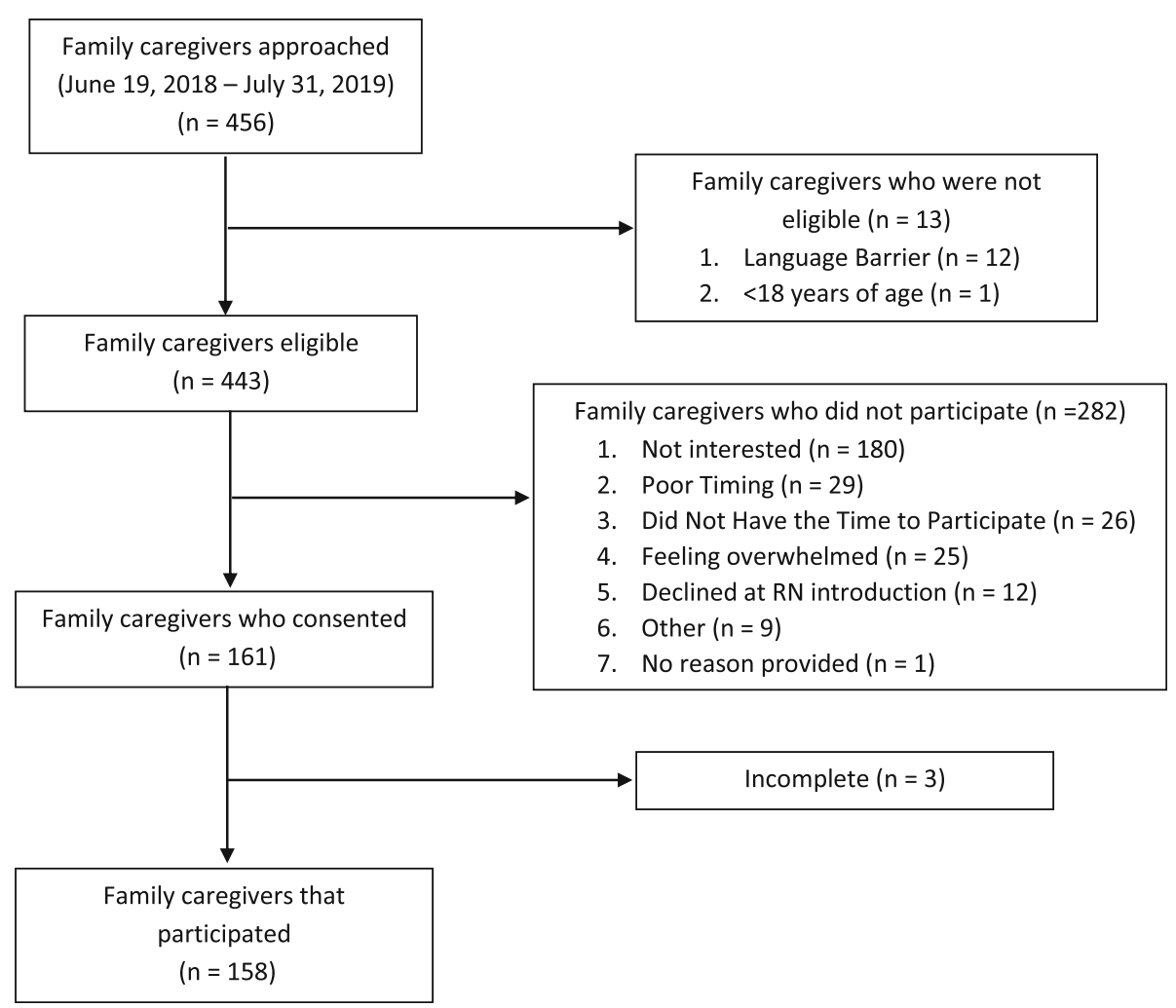

Fig. 1 Flow diagram showing family caregiver participation 
CV-ICU. The prevalence of delirium in 14 Alberta ICUs between January 2014 and June 2016 was 56.7\% (95\% Confidence Interval: 55.8-57.5) (Fiest KM, Soo, A, Lee, CH., Niven, DJ, Ely, EW, Doig, CJ, Stelfox, HT: LongTerm Outcomes of Delirium in Patients Admitted to the Intensive Care Unit: A Multi-Centre, Population-Based Cohort Study, in preparation).

We followed the Strengthening the Reporting of Observational Studies in Epidemiology (STROBE) guidelines for cross-sectional studies [18]. Inclusion criteria include: adults $\geq 18$ years of age who could provide written, informed consent and understand the English language. For the purpose of this study, a family caregiver was considered to be anyone who met this inclusion criteria. A research assistant approached eligible participants and used a script that included a standardized description of delirium. Participating family caregivers completed a paper version of a demographic questionnaire (relationship to patient, date of birth, sex, gender, ethnicity and educational background) and a paper version of the CIDKQ, both completed after enrollment in the presence of the study team.

\section{Sample size}

Sample size for the path analysis was calculated using the general rule of $10-20$ participants per estimated parameter [19]. In our path analysis, we had two regressions and four covariances, totaling six parameters. For a ratio of $10-20$ participants per estimated parameter (six), we required a sample size between 60 and 120 .

\section{Data analysis}

Descriptive statistics were examined for all study variables. Results were expressed in frequencies and percentages for categorical variables or through mean and standard deviation for continuous variables.

\section{Validity and reliability of the CIDKQ}

As there is no gold or reference standard for measuring family caregiver ICU delirium knowledge, the validity of the CIDKQ was determined through face validity and construct validity. A critical review of the CIDKQ items was discussed with a multidisciplinary group including delirium researchers, patient partners (past ICU patients and family members), ICU physicians, ICU nurses and allied health professionals. ICU nurses and allied health professionals were part of a delirium working group that meets monthly to discuss delirium initiatives. ICU physicians and patient partners are part of the department and research team, respectively. Any, additional items were discussed with the same multidisciplinary team to ensure items were simple, clear and related to ICU delirium knowledge. Due to the sample size, determination of construct validity was not possible using confirmatory factor analysis as was used in the original study of the CDKQ development [14]. Instead, we tested the model from the correlation of the three dimensions of delirium knowledge: risks (items 1-10, score range 0-10), actions (items 11-16, score range 0-6) and symptoms (items $17-21$, score range $0-5$ ). We used Pearson correlation coefficients to examine the correlation between the dimensions of delirium knowledge, wherein a Pearson $r$ value of $0.10,0.30$, and 0.50 was interpreted as small, medium, and large effect sizes, respectively [20]. We used path analysis to examine direct effects of the dimensions of delirium knowledge using maximum likelihood estimation. Path coefficients including the observed endogenous variables were calculated using the Stata package pathreg [21] with Stata version 14 (StataCorp., College Station, TX, USA).

The reliability of the CIDKQ was determined by measuring internal consistency (Cronbach's alpha). Cronbach's alpha was calculated using Stata (StataCorp, College Station, TX) wherein a correlation of

Table 1 Demographics of the Study Sample $(n=158)$

\begin{tabular}{ll}
\hline Characteristics & $\mathrm{n}(\%)$ \\
\hline Type of Family Caregiver: & \\
Child & $44(27.8)$ \\
Other & $34(21.5)$ \\
Spouse & $33(21.0)$ \\
Sibling & $29(18.3)$ \\
Parent & $18(11.4)$ \\
Education: & \\
High school or less & $52(32.9)$ \\
More than a high school & $101(63.9)$ \\
Not provided & $5(3.2)$ \\
aCultural group/race: & \\
Not provided & $70(44.3)$ \\
Caucasian/White & $32(20.3)$ \\
European & $22(13.9)$ \\
North American & $15(9.5)$ \\
Asian & $10(6.3)$ \\
First Nations & $3(1.9)$ \\
Metis & $3(1.9)$ \\
Indian/African & $2(1.2)$ \\
Black & $1(0.6)$ \\
Age, male gender & $97(61.4)$ \\
\hline IDKQ score, mean \pm standard deviation (range) & $97(61.4)$ \\
a sex & $47.6 \pm 15.2 \pm 3.4(5-21)$ \\
\hline
\end{tabular}

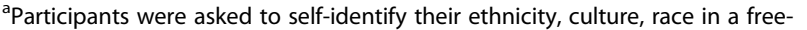
text box. They were not required to provide an answer 
0.7 was considered good and 0.8 considered excellent [22].

\section{Results}

Between June 2018 and July 2019, a total of 158 participants from the CV-ICU $(33 / 158,20.9 \%)$ and ICU $(125 / 158,79.1 \%)$ completed the CIDKQ, with a participation rate of $35.7 \%$ (158/443) (Fig. 1). Completion of the questionnaire took three to five minutes and no participant indicated it was a burden to complete. Participant characteristics are listed in Table 1. Mean participant age was 47.6 years (standard deviation [SD] 15.4 years). The majority of participants were female $(97 / 158,61.4 \%)$, adult children $(40 / 132,30.3 \%)$ of the patient. A total of 3300 answers (out of a possible 3318) were recorded for the CIDKQ, with 18 answers missing at random $(18 / 3318,0.5 \%)$. The mean score of the CIDKQ was 14.2 (SD: 3.4 , range = 2 to 21) (Table 1). The subscale means were 6.5 (SD: 1.9 , range $0-10$, possible range $0-10$ ) for the risk, 4.2 (SD: 1.3, range 0-6, possible range 0-6) for the action and 3.5 (SD: 1.2 , range $1-5$, possible range $0-5$ ) for symptom recognition subgroups. The items answered correctly by the least number of family caregivers were "If your family member had signs of sudden confusion, would you let the patient sleep during the day?" (action subscale) and items 17 and 18 of the symptom recognition subscale ("Patient

Table 2 Correctly answered items for the Caregiver ICU Delirium Knowledge Questionnaire (CIDKQ) $(n=158)$

\begin{tabular}{|c|c|c|c|}
\hline Item number & Description & Correct answer & Correctly answered, n (\%) \\
\hline \multicolumn{4}{|l|}{ Risk subgroup } \\
\hline \multicolumn{4}{|c|}{ Do you think any of the patients below might be at risk for delirium? } \\
\hline 1 & Patients who are older & Yes & $121(77.1)$ \\
\hline 2 & Patients who are married (vs. not married) & No & $74(47.4)$ \\
\hline 3 & Patients with dementia & Yes & $129(81.7)$ \\
\hline 4 & Patients with an infection & Yes & $132(84.1)$ \\
\hline 5 & Patients with more than high school education & No & $80(51.3)$ \\
\hline 6 & Patients who had recent surgery & Yes & $137(86.7)$ \\
\hline 7 & Patients who are dehydrated & Yes & $121(77.1)$ \\
\hline 8 & $\begin{array}{l}\text { Patients experiencing change in surroundings such as admission to a } \\
\text { hospital or change to another part of the hospital }\end{array}$ & Yes & $115(73.2)$ \\
\hline 9 & Patients who are mechanically ventilated or intubated & Yes & $119(75.3)$ \\
\hline 10 & Patients started on a new medication & Yes & $112(71.3)$ \\
\hline \multicolumn{4}{|c|}{ Action subgroup } \\
\hline \multicolumn{4}{|c|}{ If your family member had sign of sudden confusion, would you: } \\
\hline 11 & Orient patient to time and day and bring in photos from home & Yes & $126(80.2)$ \\
\hline 12 & Wait $24 \mathrm{~h}$ to see if the person got better & No & $82(51.9)$ \\
\hline 13 & Let the patient sleep during the day to recover & No & $49(31.8)$ \\
\hline 14 & Do nothing & No & $146(92.4)$ \\
\hline 15 & Inform the bedside RN or another member of the care team right away & Yes & $149(94.9)$ \\
\hline 16 & Ask the care team about medication changes & Yes & $137(86.7)$ \\
\hline \multicolumn{4}{|c|}{ Symptom subgroup } \\
\hline \multicolumn{4}{|c|}{ Do you think any of the patients described below might have delirium? } \\
\hline 17 & $\begin{array}{l}\text { Patients slowly becomes more confused over a few months is forgetful } \\
\text { has trouble paying attention and is more confused later in the day }\end{array}$ & No & $38(24.0)$ \\
\hline 18 & $\begin{array}{l}\text { Patients slowly becomes more confused over a few months is forgetful has } \\
\text { trouble paying attention and later in the day sees things that are not there }\end{array}$ & No & $19(12.1)$ \\
\hline 19 & $\begin{array}{l}\text { Patients suddenly becomes confused over a few days or hours floats in and out of } \\
\text { confusion during the day has trouble paying attention sees things that are no there }\end{array}$ & Yes & $138(87.3)$ \\
\hline 20 & $\begin{array}{l}\text { Patients suddenly becomes confused over a few days or hours has trouble } \\
\text { paying attention and sleeps more during the day }\end{array}$ & Yes & $116(73.9)$ \\
\hline 21 & $\begin{array}{l}\text { Patients becomes more confused over a few days and suddenly has trouble } \\
\text { getting to the bathroom on time }\end{array}$ & Yes & $93(59.2)$ \\
\hline
\end{tabular}


slowly becomes more confused over a few months (prior to ICU admission) ..." )(Table 2).

\section{Validity}

Based on the ICU delirium literature and ICU delirium experts' recommendations, several changes were made to the CDKQ to adapt it for the ICU setting (Additional file 1: Table S1). The wording "older adult" in the CDKQ was changed to "patients" for the CIDKQ. For the risk subgroup, the age for increased delirium risk was changed from "adults older than 70" to "patients who are older." The question about race or biological sex as a risk for delirium were removed because there is no consensus in the ICU delirium literature associating either with increased ICU delirium risk [15]. Lastly, an additional risk factor was added: increased delirium risk in patients who are mechanically ventilated $[15,23]$. The action subgroup included additional questions suggested by the authors of the CDKQ ("Orient patient to time of day and bring in photos from home" and "Ask the care team about medication changes"), which are supported by a narrative review on non-pharmacologic treatment and medication minimization [24]. One additional item, "Let the patient sleep during the day to recover" was added to encourage family caregivers to assist with a day/night routine as a non-pharmacological strategy to prevent or manage

a)

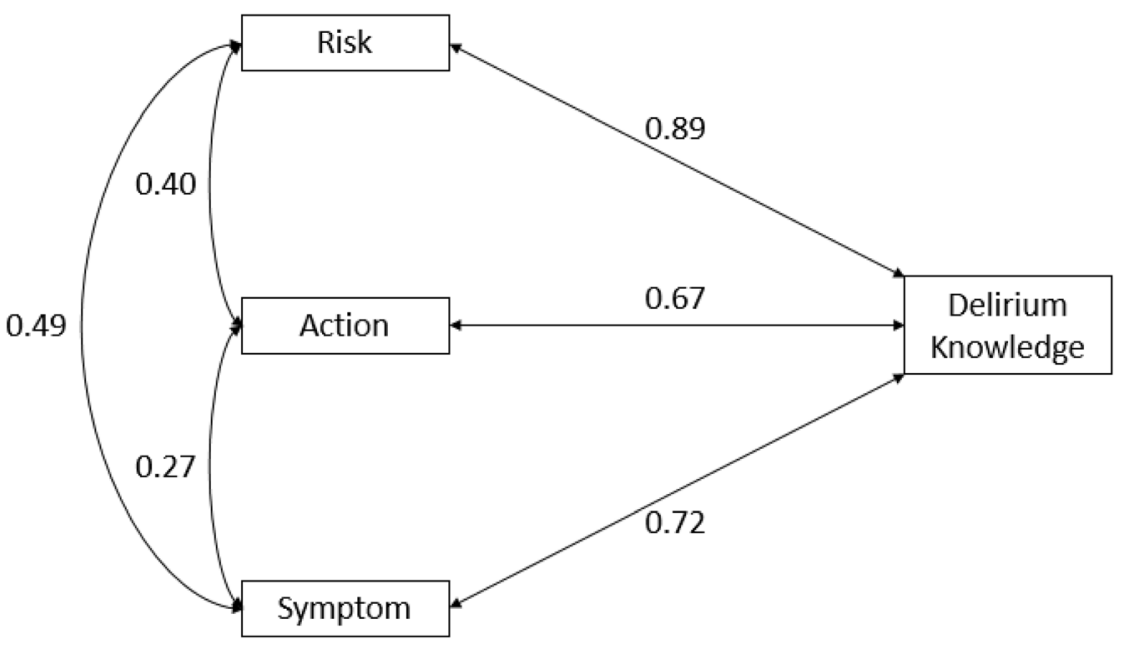

b)

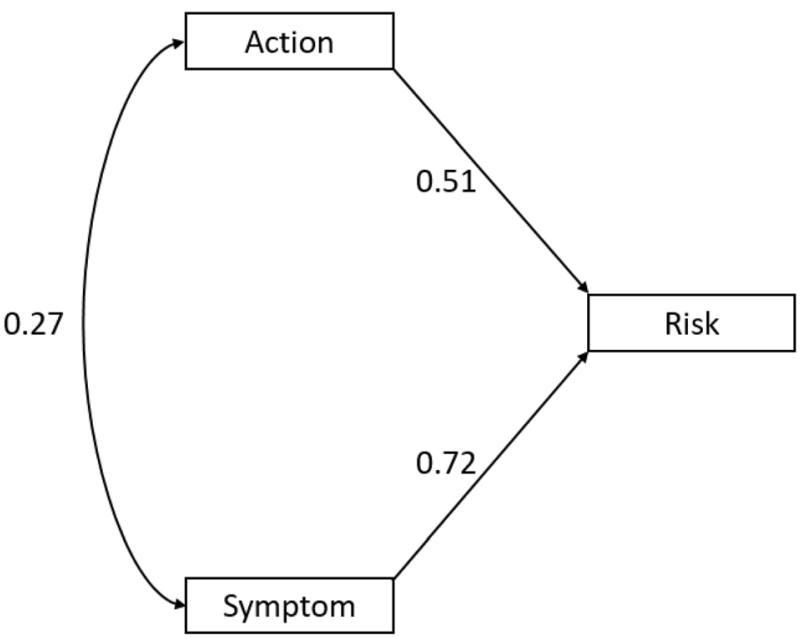

Fig. 2 a Pearson correlation coefficients between each of the three dimensions of delirium knowledge (risk, actions and symptoms) and delirium knowledge (represented by total Caregiver ICU Delirium Knowledge Questionnaire score). b Proposed pathway between the dimensions of delirium knowledge (risk, actions and symptoms). Single- and double-headed arrows represent directional effects from one variable to another and correlations between variables, respectively. Numbers on each arrow represent the Pearson correlation coefficient (double-headed arrow) and standardized path coefficient (single-headed arrow) 
delirium. No items were changed in the symptoms subgroup as they represent a range of ICU patients and address dementia versus delirium. There was 100\% agreement from the experts on the content and placement of the additional questionnaire items. Members of the multidisciplinary team found all questionnaire items were simple, clear and related to ICU delirium knowledge.

Figure 2 shows the Pearson correlation coefficients between each dimension of delirium knowledge and the proposed pathway that had the best fit between the three dimensions of delirium knowledge (risks, actions and symptoms). The correlations ranged from small (0.27, correlation between the risk and action dimensions) to medium ( 0.40 and 0.49 between risk and action dimension and action and symptom dimension, respectively). All correlations between the risk, action or symptom dimension and total CIDKQ score were considered large. An increase in total action and total symptom scores are significantly associated with an increase in total risk score (Fig. 2).

\section{Reliability}

Cronbach's alpha for internal consistency of the CIDKQ was 0.79 for the total scale, which indicates the CIDKQ has good reliability. Cronbach's alpha were 0.77 for the risk subscale, 0.43 for the action subscale and 0.57 for the symptom recognition subscale. Examination of the Cronbach's alpha for each item indicates that the total Cronbach's alpha did not change if any items were deleted from the CIDKQ (Table 3). However, items 16 and 18 had have a low correlation with the total score. If both items are removed, the Cronbach's alpha is 0.80 .

\section{Discussion}

ICU nurses evaluating a family caregiver's delirium knowledge and educating family caregiver's based on their knowledge gaps is a vital step for family caregivers to partner effectively in the prevention and management of delirium. This study demonstrated that the CIDKQ is a valid and reliable tool to measure a family caregiver's knowledge of the three dimensions of ICU delirium knowledge:

Table 3 Item analysis for the family caregiver ICU delirium knowledge questionnaire

\begin{tabular}{|c|c|c|c|}
\hline Item & Description & $\begin{array}{l}\text { Alpha if item } \\
\text { deleted }\end{array}$ & $\begin{array}{l}\text { Item-to-total score } \\
\text { correlations }\end{array}$ \\
\hline 1 & Patients with an infection & 0.78 & 0.54 \\
\hline 2 & Patients who are married (vs not married) & 0.80 & 0.33 \\
\hline 3 & Patients with dementia & 0.78 & 0.54 \\
\hline 4 & Patients who are older & 0.78 & 0.56 \\
\hline 5 & Patients with more than a high school education & 0.79 & 0.37 \\
\hline 6 & Patients who had recent surgery & 0.78 & 0.61 \\
\hline 7 & Patients who are dehydrated & 0.77 & 0.70 \\
\hline 8 & $\begin{array}{l}\text { Patients experiencing change in surroundings such as a recent ICU admission or move to new room } \\
\text { or transferred }\end{array}$ & 0.77 & 0.69 \\
\hline 9 & Patients started on a new medication & 0.78 & 0.51 \\
\hline 10 & Patients who are mechanically ventilated or intubated & 0.79 & 0.48 \\
\hline 11 & Orient patient to time and day or bring in photos from home & 0.79 & 0.38 \\
\hline 12 & Wait $24 \mathrm{~h}$ to see if the patient got better & 0.79 & 0.40 \\
\hline 13 & Let the patient sleep during the day to recover & 0.80 & 0.33 \\
\hline 14 & Do nothing & 0.79 & 0.32 \\
\hline 15 & Inform the bedside RN or another member of the care team right away & 0.79 & 0.39 \\
\hline${ }^{\mathrm{a}} 16$ & Ask the care team about medication changes & 0.80 & 0.23 \\
\hline 17 & $\begin{array}{l}\text { Patient slowly becomes more confused over a few months (prior to ICU admission), is forgetful, has } \\
\text { trouble paying attention, and is more confused later in the day }\end{array}$ & 0.80 & 0.30 \\
\hline${ }^{\mathrm{a}} 18$ & $\begin{array}{l}\text { Patient slowly becomes more confused over a few months (prior to ICU admission), is forgetful, has } \\
\text { trouble paying attention and later in the day sees things that are not there }\end{array}$ & 0.80 & 0.23 \\
\hline 19 & $\begin{array}{l}\text { While in the ICU, the patient suddenly becomes confused over a few days or hours, floats in and out } \\
\text { of confusion during the day, has trouble paying attention, sees things that are not there }\end{array}$ & 0.78 & 0.56 \\
\hline 20 & $\begin{array}{l}\text { While in the ICU, the patient suddenly becomes confused over a few days or hours, has trouble paying } \\
\text { attention, and sleeps more during the day }\end{array}$ & 0.78 & 0.49 \\
\hline 21 & $\begin{array}{l}\text { While in the ICU, the patient becomes more confused over a few days and suddenly has trouble } \\
\text { getting to the bathroom on time }\end{array}$ & 0.79 & 0.43 \\
\hline
\end{tabular}

atems with low correlation and can be removed from the CIDKQ 
risks, actions, symptoms. The content of the CIDKQ was based on relevant ICU delirium literature and consensus among a multidisciplinary team.

The reliability of the CIDKQ is comparable to the CDKQ [14]. The number of items in the risk subscale was the same $(n=10)$, but the internal consistency was better in the CIDKQ (0.77) for the risk subscale versus the CDKQ (0.66). Even though the CIDKQ actions subscale had two more items $(n=6)$ than the CDKQ $(n=4)$, the Cronbach alpha for the actions subscale was lower than the CDKQ (0.43 versus 0.72$)$. The symptom subscale had the same number of items and a higher Cronbach alpha (0.57) compared to the CDKQ symptom subscale (0.49). Items in the CIDKQ include with low correlation are items 16 and 18, which include "Ask the care team about medication changes" and description of a patient with dementia. As there is already a dementia question (item 17), item 18 can be removed. Although item 16 has low correlation with the overall score, it should remain because it empowers a family caregiver to ask the care team about possible deliriogenic medications such as benzodiazepines or prompt a discussion with the care team about their loved one's altered cognition which may be secondary to analgesia or sedation administration (i.e. not delirium). Further refinement of the CIDKQ could include additional risk factors such as patients with hypertension, patients in a coma [15] or sleep disturbance [25]. Additional items added to the actions and symptoms subscales, which may improve the reliability and the fit indices, include non-pharmacological interventions from the current delirium guidelines [13] such as "bring in eyeglasses or hearing aids" for the action subscale and an item describing a patient with agitation for the symptom subscale.

The CIDKQ has applicability in clinical practice. First, it allows for ICU nurses to assess a family caregiver's knowledge of delirium risk factors, appropriate actions when a patient has delirium and delirium symptoms and if they can distinguish delirium from dementia. As such, the ICU nurse can target delirium education to the knowledge gaps and empower the family caregiver to partner effectively to prevent and manage delirium. Family caregivers are an underutilized resource and are well-positioned to partner in prevention and management delirium. Family caregivers know the patient the best and can relate to the patient in a way that an unfamiliar member of the healthcare team cannot. This includes applying nonpharmacological strategies tailored to the patient's known preferences or calming a patient who is agitated. A recent systematic review reports that family caregiver involvement in delirium may reduce a family caregiver's anxiety and reduces a patient with delirium length of hospital stay [26]. In addition, most physicians and nurses believe families can assist with delirium prevention [27] and do not view providing delirium education to family caregivers as onerous [28]. Family caregivers are accepting of providing interventions to patients with delirium [26].

\section{Limitations}

The current study followed established cross-sectional study reporting guidelines and included a multidisciplinary team. However, there are still a few limitations. The study was conducted in two ICUs located within a single centre hospital. FMC has a catchment area of 1.5 million and includes a diverse population. On patient admission, the sampled ICU provides a pamphlet on delirium (with information on delirium risk factors, actions and symptoms), regularly screens for delirium using the Intensive Care Delirium Screening Checklist [29] and reports delirium as part of daily, multidisciplinary rounds, which family caregivers are invited to participate in. As such, family caregivers in this sample may have better delirium knowledge and these results may not be generalizable to family caregivers from other ICUs.

\section{Conclusions}

The CIDKQ is a valid and reliable measure of delirium knowledge of family caregivers of critically ill patients. This measure can help ICU nurses and researchers identify the need for delirium education in family caregivers of critically ill patients and evaluate the effectiveness of that education. The CIDKQ can be adapted for other ICU settings and updated and re-validated to align with future guideline updates.

\section{Supplementary information}

Supplementary information accompanies this paper at https://doi.org/10. 1186/s12913-020-4892-5.

Additional file 1: Table S1. Comparison of the items of the Caregiver Delirium Knowledge Questionnaire (CDKQ) and Caregiver ICU Delirium Knowledge Questionnaire (CIDKQ)

\section{Abbreviations}

CDKQ: Caregiver Delirium Knowledge Questionnaire: CIDKQ: Caregiver ICU Delirium Knowledge Questionnaire; CV-ICU: Cardiovascular Intensive Care Unit; ICU: Intensive Care Unit; PICS: Post-Intensive Care Syndrome

\section{Acknowledgements \\ The authors thank Bonnie Sept, one of the patient partners, for her help in reviewing study materials. The authors also thank Henry Ma, Katherine Liu and Israt Yasmeen for their help in enrolling participants. This study was supported by a grant to Henry T. Stelfox from the Canadian Institutes of Health Research.}

\section{Authors' contributions}

All authors made substantial contributions to this work. KDK, HTS and KMF designed the study, KDK facilitated acquisition of data and performed the data analysis; KDK, MJB, HTS, EWE and KMF interpreted the data; KDK drafted the manuscript; and all authors critically revised the manuscript and approved the final version for submission. The authors have no conflicts of interest relevant to this manuscript. 


\section{Funding}

This work was supported by a Canadian Institutes of Health Research Grant (to HTS). The funders had no role in study design, data collection and analysis, decision to publish, or preparation of the manuscript.

\section{Availability of data and materials}

The questionnaire and data used during the current study are available from the corresponding author (KMF) on reasonable request.

\section{Ethics approval and consent to participate}

The study was approved by the Conjoint Health Research Ethics Board at the University of Calgary (Reference number: REB18-0331). Written informed consent was acquired for each participant.

\section{Consent for publication}

Not applicable.

\section{Competing interests}

The authors declare that they have no competing interests.

\section{Author details}

'Department of Critical Care Medicine, Alberta Health Services \& University of Calgary, Calgary, AB, Canada. ${ }^{2}$ College of Nursing, Marquette University, Milwaukee, WI, USA. ${ }^{3}$ Tennessee Valley Veteran's Affairs Geriatric Research Education Clinical Center (VA GRECC), Critical Illness, Brain Dysfunction, and Survivorship (CIBS) Center, Vanderbilt University Medical Center, Nashville, TN, USA. ${ }^{4}$ Department of Community Health Sciences and O'Brien Institute for Public Health, University of Calgary, Calgary, AB, Canada. ${ }^{5}$ Department of Psychiatry \& Hotchkiss Brain Institute, Cumming School of Medicine, University of Calgary, Calgary, AB, Canada.

Received: 20 September 2019 Accepted: 6 January 2020

Published online: 14 February 2020

\section{References}

1. Ely EW, Shintani A, Truman B, Speroff T, Gordon SM, Harrell FE, et al. Delirium as a predictor of mortality in mechanically ventilated patients in the intensive care unit. JAMA. 2004;291:1753-62.

2. Brummel NE, Jackson JC, Pandharipande PP, Thompson JL, Shintani AK, Dittus RS, et al. Delirium in the ICU and subsequent long-term disability among survivors of mechanical ventilation. Crit Care Med. 2014;42(2):36977.

3. Pandharipande PP, Girard TD, Jackson JC, Morandi A, Thompson JL, Pun BT, et al. Long-term cognitive impairment after critical illness. N Engl J Med. 2013;369(14):1306-16.

4. Rawal G, Yadav S, Kumar R. Post-intensive care syndrome: an overview. J Transl Int Med. 2017:5(2):90-2.

5. Partridge JS, Martin FC, Harari D, Dhesi JK. The delirium experience: what is the effect on patients, relatives and staff and what can be done to modify this? Int J Geriatr Psychiatry. 2013;28(8):804-12.

6. Schmitt EM, Gallagher J, Albuquerque A, Tabloski P, Lee HJ, Gleason L, et al. Perspectives on the delirium experience and its burden: common themes among older patients, their family caregivers, and nurses. Gerontologist. 2019:59(2):327-37.

7. Finucane AM, Lugton J, Kennedy C, Spiller JA. The experiences of caregivers of patients with delirium, and their role in its management in palliative care settings: an integrative literature review. Psychooncology. 2017;26(3):291300.

8. Racine AM, D'Aquila M, Schmitt EM, Gallagher J, Marcantonio ER, Jones RN, et al. Delirium burden in patients and family caregivers: development and testing of new instruments. Gerontologist. 2018. https://doi.org/10.1093/ geront/gny041.

9. Desai SV, Law TJ, Needham DM. Long-term complications of critical care. Crit Care Med. 2011;39(2):371-9.

10. Needham DM, Davidson J, Cohen H, Hopkins RO, Weinert C, Wunsch $H_{\text {, }}$ et al. Improving long-term outcomes after discharge from intensive care unit: report from a stakeholders' conference. Crit Care Med. 2012;40(2):5029.

11. Davidson JE, Jones C, Bienvenu OJ. Family response to critical illness: postintensive care syndrome-family. Crit Care Med. 2012;40(2):618-24.
12. Davidson JE, Aslakson RA, Long AC, Puntillo KA, Kross EK, Hart J, et al. Guidelines for family-centered Care in the Neonatal, pediatric, and adult ICU. Crit Care Med. 2017:45(1):103-28.

13. Devlin JW, Skrobik Y, Gelinas C, Needham DM, Slooter AJC, Pandharipande PP, et al. Clinical practice guidelines for the prevention and management of pain, agitation/sedation, delirium, immobility, and sleep disruption in adult patients in the ICU. Crit Care Med. 2018;46(9):e825-e73.

14. Bull MJ, Avery JS, Boaz L, Oswald D. Psychometric properties of the family caregiver delirium knowledge questionnaire. Res Gerontol Nurs. 2015;8(4): 198-207.

15. Zaal IJ, Devlin JW, Peelen LM, Slooter AJ. A systematic review of risk factors for delirium in the ICU. Crit Care Med. 2015;43(1):40-7.

16. Salluh Jl, Wang H, Schneider EB, Nagaraja N, Yenokyan G, Damluji A, et al. Outcome of delirium in critically ill patients: systematic review and metaanalysis. BMJ. 2015;350:h2538.

17. Teel CS, Meek P, McNamara AM, Watson L. Perspectives unifying symptom interpretation. Image J Nurs Sch. 1997;29(2):175-81.

18. von Elm E, Altman DG, Egger M, Pocock SJ, Gotzsche PC, Vandenbroucke $J P$. The strengthening the reporting of observational studies in epidemiology (STROBE) statement: guidelines for reporting observational studies. Int J Surg. 2014;12(12):1495-9.

19. Schreiber JB. Core reporting practices in structural equation modeling. Res Soc Admin Pharm RSAP. 2008;4(2):83-97.

20. Gignac E, Szodorai ET. Effect size guidelines for individual differences researchers. Personal Individ Differ. 2016;102:74-8.

21. Ender PB. pathreg: Path analysis using ols regression. UCLA: Statistical Consulting Group 2009 [Available from: http://www.ats.ucla.edu/stat/stata/ faq/pathreg.htm.

22. Cortina JM. What is coefficient alpha? An examination of theory and applications. J Appl Psychol. 1993;78(1):98-104.

23. Ely EW, Inouye SK, Bernard GR, Gordon S, Francis J, May L, et al. Delirium in mechanically ventilated patients: validity and reliability of the confusion assessment method for the intensive care unit (CAM-ICU). JAMA. 2001; 286(21):2703-10.

24. Blair GJ, Mehmood T, Rudnick M, Kuschner WG, Barr J. Nonpharmacologic and medication minimization strategies for the prevention and treatment of ICU delirium: a narrative review. J Intensive Care Med. 2018. https://doi.org/ 10.1177/0885066618771528.

25. Flannery AH, Oyler DR, Weinhouse GL. The impact of interventions to improve sleep on delirium in the ICU: a systematic review and research framework. Crit Care Med. 2016;44(12):2231-40.

26. McKenzie J, Joy A. Family intervention improves outcomes for patients with delirium: systematic review and meta-analysis. Australas J Ageing. 2019. https://doi.org/10.1111/ajag.12688.

27. Smithburger PL, Korenoski AS, Kane-Gill SL, Alexander SA. Perceptions of family members, nurses, and physicians on involving Patients' families in delirium prevention. Crit Care Nurse. 2017;37(6):48-57.

28. Mitchell ML, Kean S, Rattray JE, Hull AM, Davis C, Murfield JE, et al. A family intervention to reduce delirium in hospitalised ICU patients: a feasibility randomised controlled trial. Intensive Crit Care Nurs. 2017:40:77-84.

29. Ouimet S, Riker R, Bergeron N, Cossette M, Kavanagh B, Skrobik Y. Subsyndromal delirium in the ICU: evidence for a disease spectrum. Intensive Care Med. 2007;33(6):1007-13.

\section{Publisher's Note}

Springer Nature remains neutral with regard to jurisdictional claims in published maps and institutional affiliations.

\section{Ready to submit your research? Choose BMC and benefit from:}

- fast, convenient online submission

- thorough peer review by experienced researchers in your field

- rapid publication on acceptance

- support for research data, including large and complex data types

- gold Open Access which fosters wider collaboration and increased citations

- maximum visibility for your research: over $100 \mathrm{M}$ website views per year

At BMC, research is always in progress.

Learn more biomedcentral.com/submissions 